\title{
AUTHOR INDEX *
}

Afanasieva, P. M. 169

Aoki, S. 123, 816, 819, 821, 816

Argue, A. N. 270, 757

Arlot, J.-E. 369

Argyle, R. W. 213

Benevides-Soares, P. 103, 565, 830

Bernacca, P.L. 820, 829

Billaud, G. 389, 395, 419

Bowell, E. 231

Breakiron, L. 341

Brosche, P. 53

Brumberg, V. A. 19

Chiumiento, G. 443

Chollet, F. 705

Clarke, R. T. 397

Clauzet, L. B. F. 103

Colavita, M. 331

Cole, C. S. 612

Corben, P. M. 213

Corbin, T. E. 75, 86, 505, 747, 778

Cudworth, K. M. 201, 208, 822

Currie, D. 504, 542, 591

Dačić, M. 571

Dahn, C. 815

Davis, M. S. 826

de Vegt, C. 121, 155, 173, 179, $208,242,299,442,591,603$, $634-635,641,763,810,825$

Débarbat, S. 481, 705, 788, 821

Di Fatta, C. 341

Douglas, G. G. 649

$\mathrm{Du}, \mathrm{M}-\mathrm{H} .551$

Duma, D. P. 243

Eichhorn, H. 11, 155, 230, 236, $251,319,603,634,641,675$, $727,738,747,755,778,826$

Elsmore, B . 121, 737, 738793

Ershov; V. N. 407

* Note: bold face numbers refer to the authors of the papers printed in this volume, ordinary numbers to discussion contributions. Numbers higher than 810 indicate pictures.
Esposito, P. B. 789

Fabricius, C. 465, 468

Fan, Y. 551

Fanselow, J. L. 163, 794, 814

Fedorov, P.N. 529

Fedorova, R. T. 529

Feo, J. 637

Florkowski, D. R. 179, 181, 817

Fomin, V. A. 169, 571

Fracastoro, M. G. 443, 829

Fresneau, A. 643, 810, 813, 824

Froeschle, M. 113

Fujishita, M. 123

Gatewood, G. D. 341, 352, 823

Gauss, F. S. 483

Gibbs, P. 497

Gliese, W. 45, 190, 199, 319, $653,667,675,719,747$

Gottesman, S. T. 817

Goto, Y. 133

Gubanov, V. S. 765

Guinot, B. 1, 12, 74, 111, 141, 257, $591,603,736,805$

Gumerov, R.I. 459

Hanson, R. B. 43, 45

Harrington, R. S. 271, 273, 287, $603,641,815,821,829$

Hartkopf, W. I. 301, 307

Heintz, W. D. 319, 651, 748

Helmer, L. 429, 433, 505

Hemenway, P. D. 251, 295, 613, $624,676,778$

Herring, T. 121, 131

Hфg, E. 340, 395, 413, 419, 426, $442,496,553,542,604,612$, $625,634,635,727$

$\mathrm{Hu}$, N. 421, 426, 469, 481

$\mathrm{Hu}, \mathrm{S}-\mathrm{S} .551$

Hughes, J. A. 330, 442, 458, $483,496,565,604,755$

Jefferys, W. H. 352, 637, 641, 805, 819

Johnston, K. J. 131, 179, 227, 331, 340,823

Kapkov, V. B. 459

Keating, R. E. 397 
Kharin, A. S. 427, 463, 685

Khrutskaya, E. V. 691

Kiewiet de Jonge, J. 341

Kizjun, L. N. 243

Klemola, A. R. 191, 199

Klock, B. L. 507, 515, 821

Konin, V. V. 569

Kovalevsky, J. 86, 121, 141,581, $591,604,793,805,820$

Kucharskij, L. A. 463

Kudeeva, V. S. 795

Kllhne, C. 379

Laptienko, N. I. 243

Lazorenko, P. F. 463

Leacock, R. J. 817

Lee, J. -F. 209, 237, 242

Lestrade, J. -F. 779

Li, D.-M. 375, 567

Li, H. 551

Li, Z.-M 551

Lieske, J. 74, 181, 763

Liewer, K. M. 163

Lindegren. L. 593, 603, 604, 830

Lopez, C. E. 209

Lukac, C. F. 397

Luo, D.-J. 375

Lutz, T. E. 47

L'vov, V. N. 169

Ma, C. 157, 162

Machado, L. E. daS. 229

Mao, W. 551

Mazurier, J. M. 435

McAlister, H. 293, 299, 300, 330

McCarthy, D. W. 309, 319, 822

Melinkov, M. A. 243

Meyer, C. 113

Mingaliev, M. G. 169

Minyajlo, N. F. 463

Mitić, L. A. 525

Miyamoto, M. 379, 449, 557, 565, 816

Morrison. L. V. 86, 102, 497, 504, 505,683

Murray, C. A. 12, 86, 199, 208, 213, 227, 270, 273, 468, 624, $641,675,727,736,805,819$, 831

Mutel, R. L. 779

Nakajima, K. 123

Newhall, X X 789, 793, 794, 818

Niell, A. E. $45,163,181,779,788$, 818,825
Noel 1, F. 715

Pakvor, I. 525

Pavlov, B. A. 795

Petrov, G. M. 517, 529

Phillips, R. B. 779

Pinigin, G.I. 459

Pliss, V. E. 407

Pogonij, A. D. 569

Pozhalov, A. A. 169

Polozhentsev, A. A. 691

Polozhentsev, A. D. 691

Preston, R. A. 779, 789

Radchuck, A. G. 795

Rafferty, T. J. 95, 102

Rapaport, M. 543

Reasenberg, R. D. 321, 330, 814

Requième, Y. 435, 442, 543, 550, 813

Robertson, D. S. 143, 155

Robinson, M. D. 483

Røser, S. 330, 634, 773, 778, 816, 831

Russell, J. L. 242, 259, 697, 703, 721,727

Sadzakov, S. 571

Safronov, Yu. I. 243

Salukvadze, G. N. 289

Sarasso, M. 443

Sato, K. 133, 141

Scargle, J. D. 353, 368

Schwan, H. 53, 63, 74, 442, 816

Sergienko, V. I. 795

Shao, M. 331, 340

Simon, R. 331

Smith, C. 505, 542, 612, 669, 675, 676

Sôma, M. 123

Sovers, O. J. 163

Staelin, D. 331

Standish, E. M. 667, 683, 818

Stein, J. 341, 822

Stepanova, E. A. 691

Stock, J. 253, 257

Stone, R. C. 483

Strand, K. Aa. 131, 190, 208, 228, $352,462,667,738,747,827$

Streletsky, Yu. S. 407

Takahashi, Y. 123

Tanikawa, K. 133

Teleki, G. 12, 426, 433, 468, 515, 542,749

Thomas, J. B. 163 
Thornburg, J. 352, 591, 603, 641, $703,738,794$

Thuillot, W. 369

Townes, C. 591,788

Treuhaft, R. N. 163

Tsay, W. -S. 237

Tsesis, M. L. 463

Turon-Lacarrieu, C. 605, 612, 812, 828

Upgren, A. R. 190, 247, 251, 273, 818

van Altena, W. F. 183, 190, 208, 209 , 237, 251, 273, 352, 550, $612,651,812,828$

Vityazev, V. V. 87

Wackernagel, H. -B. 819, 822, 827

Wade, C. M. 179

Wallace, K. S. 163

Walter, H. G. 162, 223, 227, 228, $612,788,794,812,831$

Wan, L. 275

Wang, L. -j. 275

Warren, W. 300, 739, 747, 824

Wasserman, L. 231, 236, 815

Westerhout, G. 131, 727, 738, 747, $799,805,828$

Wheeler, P. J. 379

Williams, C. A. 259, 270

Worley, C. E. 649, 651, 815, 822, 824

$\mathrm{Xu}, \mathrm{B} . \mathrm{X} .13$

Yazudin, L. I. 691

Yang, Z. -h. 275

Ye, S.-h. 11, 208, 442, 651, 729, 736

Yokoyama, K. 133

Yoshizawa, M. 379, 449, 458, 557

Zhang, H. 13

Zheng, Y. -j. 275

Zhu, N. -h. 275

Zhu, S. Y. 13

Zverev, M. Z. 691

Zverev, Yu. K. 169 